\title{
Review of: "ACE2-like carboxypeptidase B38-CAP protects from SARS-CoV-2-induced lung injury"
}

xiaojuan su

Potential competing interests: The author(s) declared that no potential competing interests exist.

This work explored the treatment potentiality of B38-CAP in SARS-CoV-2-induced lung injury. They found that B38-CAP significantly improves lung edema and pathologies of lung injury in SARS-CoV-2-infected hamsters or human ACE2 transgenic mice. This study provide the first in vivo evidence that increasing ACE2-like enzymatic activity is a potential therapeutic strategy to alleviate lung pathologies in COVID-19 patients. However, there are some concerns and shortcomings:

(1). For B38-CAP treatment, the author selected intraperitoneal injection, why chose this pathway? Please discuss. If this study aims to explore B38-CAP as a potential drug for the COVID-19 patients, how this drug transfer pathway meets with the clinical requirement? Please give detail discussion.

(2). The B38-CAP ( $2 \mathrm{mg} / \mathrm{kg} /$ day) was injected once a day after $12 \mathrm{~h}$ of post infection, how only once injection could bring such good effect? In addition, $12 \mathrm{~h}$ of post infection was o short treatment window for clinical use as we know the COVID-19 patients mostly validated until the phenotype appears. So, did the author had done some condition selection experiments for the injection dose, injection time and injection times? Besides, whether B38-CAP injection with some potential side effects $\square$ Please add additional experiments to indicate these questions or give detail discussion. 